\title{
Cosmic multi-muon bundles detected by DELPHI
}

\author{
Jan Řídký, Petr Trávníček \\ Institute of Physics, Academy of Sciences of the Czech Republic
}

\begin{abstract}
The DELPHI detector at LEP, located $100 m$ underground, has been used to detect multi-muon bundles originating from cosmic ray interactions in the atmosphere. The cosmic events registered during the years 1999 and 2000 correspond roughly to $1.610^{6} s$ of effective run time. The aim of the work is to compare predictions of high energy interaction models and the measured muon multiplicity distributions and also to determine the absolute flux of events in certain muon multiplicity range. The presentation describes the current status of the analysis.
\end{abstract}

Contributed Paper for ICHEP 2002 (Amsterdam) 


\section{Introduction}

The cosmic rays bombarding the Earth atmosphere span an enormous range of energy and they carry information about various processes in Solar system, our Galaxy and the outer space. The chemical composition of cosmic rays is known from direct measurements by balloon and satellite based experiments which detect cosmic particles in the upper part of atmosphere (RUNJOB, JACEE, ...). Because the cosmic ray flux is rapidly falling with increasing energy, limited detection area and exposure time of these experiments do not allow us the direct detection of cosmic rays at energies higher then $10^{14} \mathrm{eV}$.

The information on cosmic rays at higher energies is obtained indirectly from the ground and underground experiments. The aim of these experiments is to explore the cosmic ray energy spectrum and to understand the mass composition of primary cosmic particles, especially in the energy range $10^{15}-10^{18} \mathrm{eV}$, so called 'knee' region, where the spectral index of the energy spectrum changes. The ground experiments use large arrays to detect extensive cosmic ray showers at energies extending orders of magnitudes above $10^{14} \mathrm{eV}$ (KASCADE, EASTOP, ... ). ${ }^{1}$ In order to extract maximum possible information on individual showers the technologically most advanced arrays detect separately their electromagnetic, muon and in lesser extent also the hadron component [1]. The muon component of atmospheric showers, initiated by cosmic rays interacting in the upper atmosphere, has been subject of interest for many experiments. The underground experiments in fact detect only the muon component, but contrary to the surface experiments, they detect the hard component of the spectrum with momentum cutoff imposed by the thickness of the overburden. Most of the underground experiments are former proton decay experiments located so deep underground that the muon momentum cutoff is of the order of $\mathrm{TeV}$ or at least several hundreds $\mathrm{GeV}$ and only few muons penetrate to the detector $([2],[3],[4],[5],[6],[7])$. The few experiments with medium overburden have either crude spatial resolution like the underwater experiments [8] and [9] or they have small detection area like [10] $\left(6 \times 16 \mathrm{~m}^{2}\right)$. The data presented here were recorded by the DELPHI experiment (CERN) at an intermediate underground depth $(100 \mathrm{~m})$ corresponding to energy cutoff for vertical muons $\sim 50 \mathrm{GeV}$. The events were detected by cathode readout of DELPHI hadron calorimeter with good spatial resolution and with DELPHI tracking detectors TPC and barrel muon chambers. Thus the collected data help to fill the gap in overall data on extensive air showers.

When dealing with data on muons with energies exceeding $\sim 50 \mathrm{GeV}$, the estimation of primary particle energy and especially the mass composition of the incoming particles are linked to the dynamics of the first interactions in the course of the shower development. The Monte Carlo models which simulate the nucleus-nucleus interaction are used to correlate measured quantities with primary particle energy and type. There are several Monte Carlo models ( NEXUS, QGSJET [11], SIBYLL, ...) used to describe extensive air showers. These models are tuned to data at energies accessible by accelerator experiments which are much lower. However, the cosmic ray energy spectrum is well established and the agreement between several experiments has been obtained. On the other hand, the estimation of cosmic ray chemical composition is much more model dependent and therefore more difficult [12]. Even if large progress has been made in this field, (KASKADE composition results [13]) and new results are expected, it is clear that the models should

\footnotetext{
${ }^{1}$ We shall not discuss the experiments based on the fluorescence technique of cosmic ray detection as it does not reconstruct individual particles in showers
} 
be tuned to as many measurements as possible.

The DELPHI detector at LEP has been used to detect the cosmic multi-muon bundles by hadron calorimeter with fine granularity and by tracking detectors (TPC, muon chambers). The cosmic events registered during the years 1999 and 2000 correspond roughly to $1.610^{6} \mathrm{~s}$ of effective run time. Our aim is to compare predictions of the high energy interaction models with the measured muon multiplicity distributions at momentum cutoff imposed by the overburden of the DELPHI underground experimental area ( $\geq 50 \mathrm{GeV})$. This conditions allow us to detect muons originating from primary particles with energies $10^{14}-10^{17} \mathrm{eV}$.

The importance to study the relevance of interaction models at intermediate underground depth was stressed in the work [15] by the COSMO-ALEPH collaboration. Their results indicate the excess of high multiplicity events when compared to MC simulation. Consequently the independent qualitative cross-check of this interesting result is one of the aims of our analysis.

This presentation describes the current stage of the work, ie. DELPHI measurement of muon multiplicity distributions and comparison between the data and MC prediction of QGSJET model. This model is widely used by different experiments and it extrapolates lower energy dependencies to higher energies (up to $\sim 10^{20} \mathrm{eV}$ ). Consequently the consistency check with the measurable quantity at intermediate energy range is very important. 


\section{Trigger conditions and run selection}

To detect the cosmic events, DELPHI cosmic trigger (DCT) has been implemented to the DELPHI trigger system. It requires 3 active time of flight (TOF) detector sectors ( TOF MJ3 ) to trigger the event. Data has been taken from the 'STIC' stream, where all events with active DCT trigger have been recorded among the events of Bhabha scattering. At the first stage of analysis all events with at least one track detected by HCAL cathode readout in the barrel region have been selected. The track was defined as at least 4 HCAL cathode hits in a line extended over at least $50 \mathrm{~cm}$ in $(x, y)$ projection in DELPHI frame. The event rate has been compared for different time periods and the run dependent behaviour of the rate has been observed. For the calculation of the event rate it is necessary to use the live time obtained according to number of active beam cross-overs (BCOs). It automatically corrects 2 times higher rate for data taken in 8 bunch mode compared to results obtained during 4 bunch period. Live times of runs with 8 bunches in the machine are thus effectively transformed to 4 bunch regime.

At least 4 muons $\left(N_{\mu}>3\right)$ are required to be detected by the HCAL cathode readout in order to ensure 3 TOF counters to be hit by muons and to avoid the noise dependent trigger decision. These tracks have to be furthermore parallel within $5 \mathrm{deg}$ in $(x, y)$ projection and at least one of these tracks has to have perigee $\geq 50 \mathrm{~cm}$. For multiplicity higher than 3 almost all events are triggered by DCT (99.4\%). Tab. 1 shows 14 main types of trigger conditions together with corresponding number of runs and total live times. Triggers with total live times less than $10^{5} \mathrm{~s}$ were also studied but results were strongly influenced by statistical fluctuations. The number of events lost in these short trigger periods represents about $9 \%$ of the total number of events.

Comment $(*)$ in Tab. 1 marks the problems during the beginning of the year 1999. Timing problems occurred when running at simulated frequency of 8 bunch mode for cosmics in 1999 and the DCT was not put automatically to the trigger settings for next two trigger periods. Comment $\left({ }^{* *}\right)$ means the DCT is not in the corresponding lookup table. Events are thus triggered by different detectors (e.g. TPC, ID, OD ...).

Fig. 1 plots the event rate $\left(N_{\mu}>3\right)$ for different trigger settings. The event rates are consistent within their statistical errors for all the triggers when DCT was plugged in correctly and permanently. The dependence of event rates on the run number is the result of the DCT presence (or non-presence) in the trigger setting. Thus only the runs with DCT correctly running are used for further analysis. The total live time of selected trigger / run periods is $8.6610^{6} \mathrm{~s}$. The distribution of event arrival times from TOF is constant up to $4 \mu s$ after the BCO and vanishes quickly for larger arrival times. Consequently the effective data taking time is about $100 \times 4 / 22.23=18 \%$ of the total live time, ie. $T_{\text {eff }}=1.610^{6} \mathrm{~s}$.

The agreement of event rates between different triggers was studied also as a function of muon multiplicity measured in HCAL. The sufficient agreement is obtained for all multiplicities starting from 4 and higher.

The disagreement for smaller multiplicities could be explained by noise dependence of the trigger. In the ideal situation only two muons in TOF are enough to hit 3 TOF sectors and thus activate the DCT trigger. However, the TOF detection efficiency together with possible noise in the different TOF counters may either decrease the trigger efficiency or to fake false triggers at small multiplicities. Thus for small multiplicities the efficiency of event to be triggered depends more on the actual state of TOF than for events with 
bigger multiplicities.

As an example two trigger settings (index 4 and 7) are compared with the trigger 12 (reference trigger). Fig. 2 shows the quantity $\mathrm{R}$ as a function of muon multiplicity $N_{\mu}$. The quantity $\mathrm{R}$ is defined as:

$$
R=\frac{N\left(T E S T, N_{\mu}\right) \cdot T_{R E F}}{N\left(R E F, N_{\mu}\right) \cdot T_{T E S T}}
$$

where $N\left(T E S T / R E F, N_{\mu}\right)$ is the number of events with muon multiplicity $N^{\mu}$ for studied/reference trigger and $T_{T E S T} / T_{R E F}$ are the total run times of the triggers. $\mathrm{R}$ should be essentially 1 for perfect agreement between tested and reference data. Staring from multiplicity 4 the agreement is obtained for the trigger index 7 . For the trigger index 4 the agreement is attained even for smaller multiplicities. Similar results are obtained for all the remaining triggers with DCT active.

\begin{tabular}{|c|c|c|c|c|c|c|}
\hline$I$ & PY1/PY2 & $N_{R U N}$ & $t[s]$ & year & run type & comment \\
\hline 1 & $6 / 5$ & 136 & $1.0310^{6}$ & 1999 & $\mathrm{C}$ & $*$ \\
\hline 2 & $5 / 5$ & 409 & $1.6410^{6}$ & 1999 & $\mathrm{P}$ & $*$ \\
\hline 3 & $10 / 5$ & 52 & $0.1510^{6}$ & 1999 & $\mathrm{P}$ & $*$ \\
\hline 4 & $18 / 6$ & 511 & $1.9710^{6}$ & 1999 & $\mathrm{P}$ & \\
\hline 5 & $19 / 11$ & 36 & $0.1410^{6}$ & 1999 & $\mathrm{P}$ & \\
\hline 6 & $20 / 6$ & 11 & $0.1310^{6}$ & 2000 & $\mathrm{C}$ & \\
\hline 7 & $21 / 6$ & 127 & $2.2710^{6}$ & 2000 & $\mathrm{C}$ & \\
\hline 8 & $18 / 6$ & 68 & $0.3410^{6}$ & 2000 & $\mathrm{P}$ & \\
\hline 9 & $23 / 17$ & 57 & $0.2310^{6}$ & 2000 & $\mathrm{P}$ & \\
\hline 10 & $25 / 18$ & 364 & $1.1310^{6}$ & 2000 & $\mathrm{P}$ & $* *$ \\
\hline 11 & $31 / 19$ & 319 & $1.0410^{6}$ & 2000 & $\mathrm{P}$ & \\
\hline 12 & $33 / 19$ & 371 & $1.1110^{6}$ & 2000 & $\mathrm{P}$ & \\
\hline 13 & $33 / 20$ & 530 & $1.4310^{6}$ & 2000 & $\mathrm{P}$ & \\
\hline 14 & $34 / 20$ & 397 & $1.2610^{6}$ & 2000 & $\mathrm{P}$ & $* *$ \\
\hline
\end{tabular}

Table 1: Trigger settings with live times larger then $10^{5} \mathrm{~s} . I$ is the trigger label. Numbers in $P Y 1 / P Y 2$ column represent the Pythia lookup tables for first and second level of the trigger. Time $t$ is the total life time of corresponding $N_{R U N}$ runs. Label $C$ denotes dedicated cosmic run and $P$ denotes the ordinary $e^{+} e^{-}$run. Comments $\left(^{*}\right)$ and $\left({ }^{* *}\right)$ are explained in the text. 


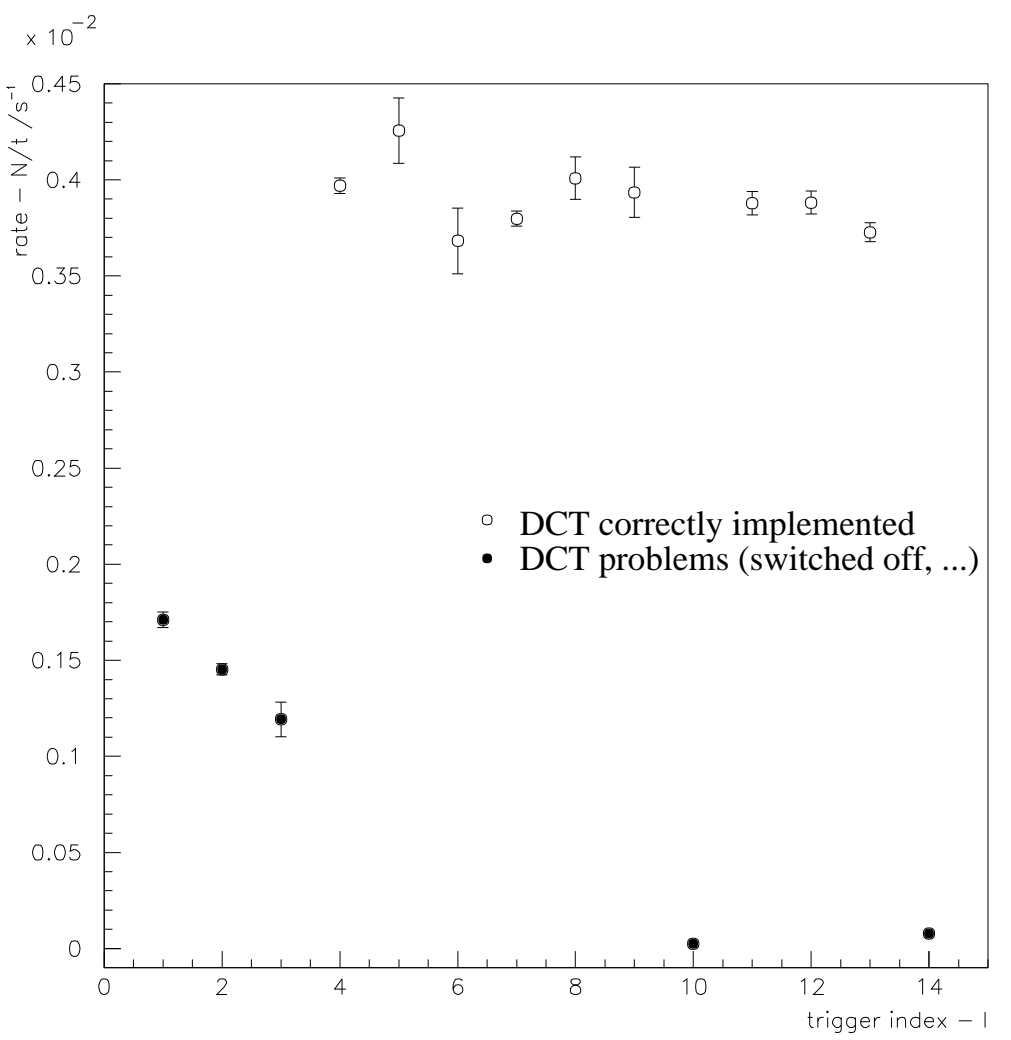

Figure 1: Event rates $\left(N_{\mu}>3\right)$ for different trigger settings.

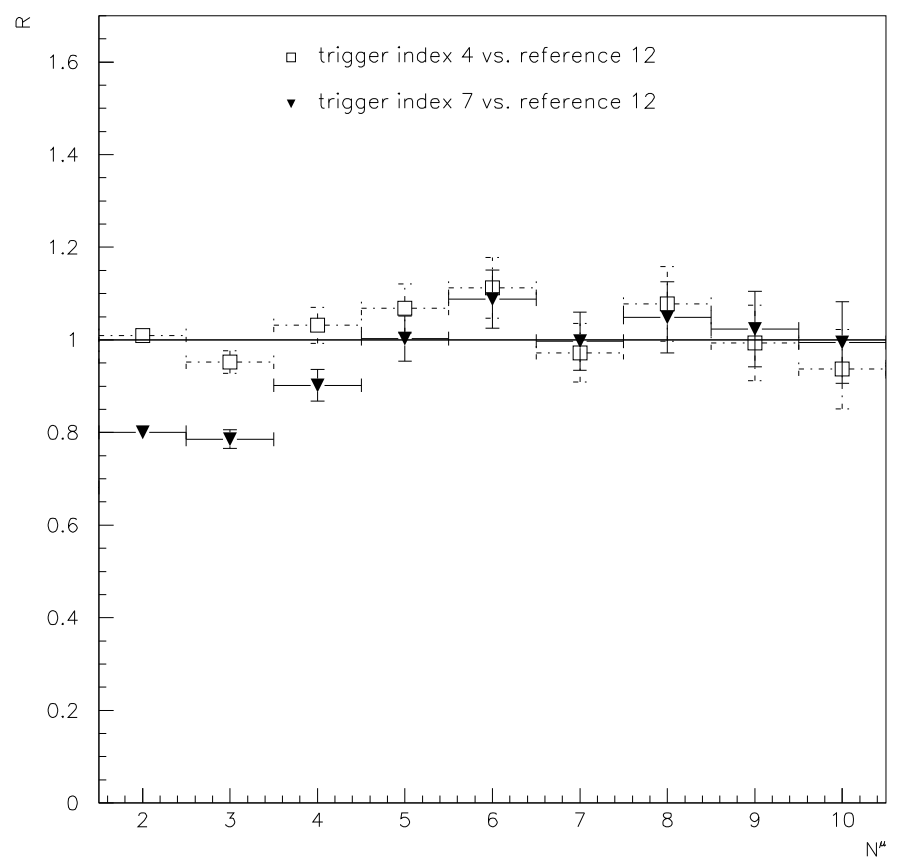

Figure 2: Dependence of the quantity $R$ defined in the text on the muon multiplicity $N^{\mu}$. Only the events that are triggered by DCT are taken into account. 


\section{Data}

ECTANA reconstruction package ([14]) is applied to reconstruct muon tracks from HCAL cathode readout in all selected runs. The minimal track length of $50 \mathrm{~cm}$ is required. No explicit assumption of track parallelism is used in the reconstruction. Fig. 3 shows the effective detection area of HCAL as a function of azimuthal angle of incoming shower. The area is similar in size with the KGF experiment [10].

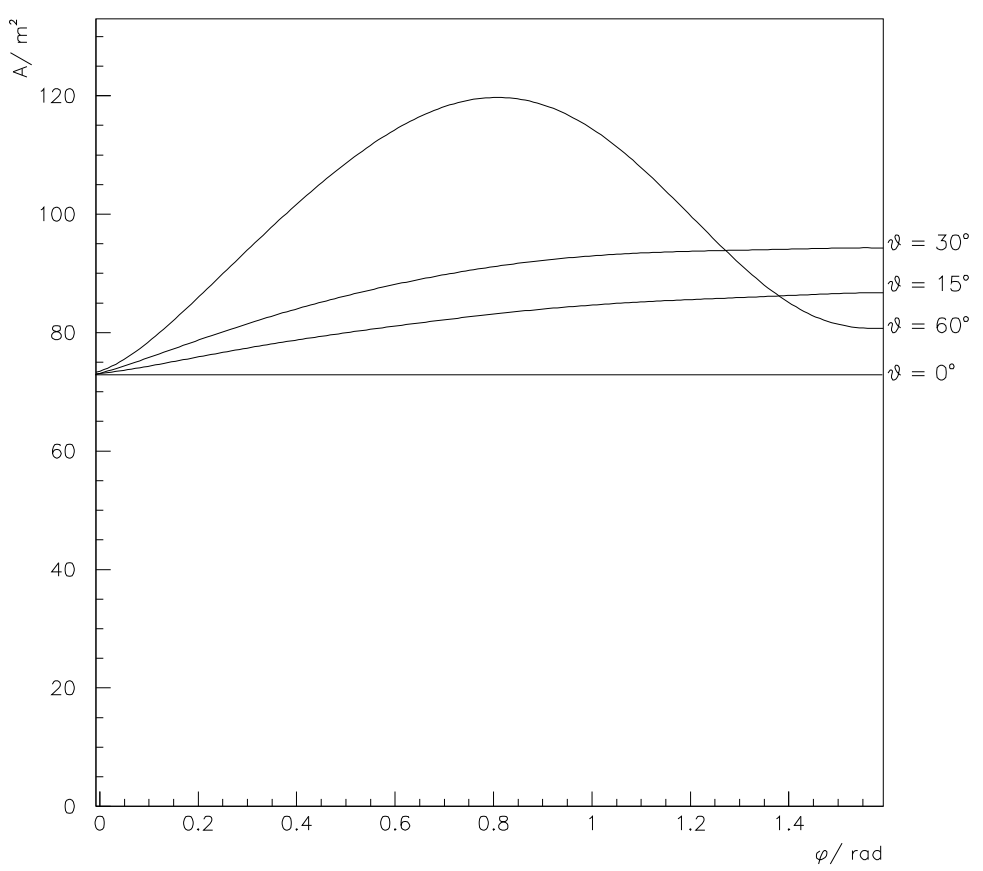

Figure 3: The effective detection area of HCAL as a function of azimuthal angle for different zenith angles. the tracks selection criteria are taken into account

The data sample consists of 89991 events with the muon multiplicity higher than 3 $\left(N_{\mu}>3\right)$. The number of events with at least 30 muons in the apparatus is 1065 .

The parallelism of reconstructed tracks was checked according to the cut that requires more than $50 \%$ of reconstructed tracks to be be within $5^{\circ}$ from the mean value of all track angles in the event. The cut leads to 14 rejected events with multiplicity higher than 30. The manual event scanning has been also performed $\left(N_{\mu} \geq 30\right)$ in order to check and ensure the rejection of electromagnetic showers originating from muon interactions in the cavern ceiling or detector material. These showers in principle could be taken as multi-muon events if no condition on track parallelism is required or no scanning is performed.

Distributions of reconstructed track angles show narrow peaks $\left(\sigma \sim 2^{\circ}\right)$ around the central values of each event. This procedure defines the average angle corresponding to incoming shower direction, (for example see event with $\sim 120$ muons in Fig. 4). The distribution of reconstructed shower angles is plotted in Fig. 5 for all events with more then 4 reconstructed muons. Authors of the work [15] found the $\cos ^{n} \theta(n=3.32)$ dependence of the zenith angle distribution. The dashed line in the Fig. 5 represents the corresponding 


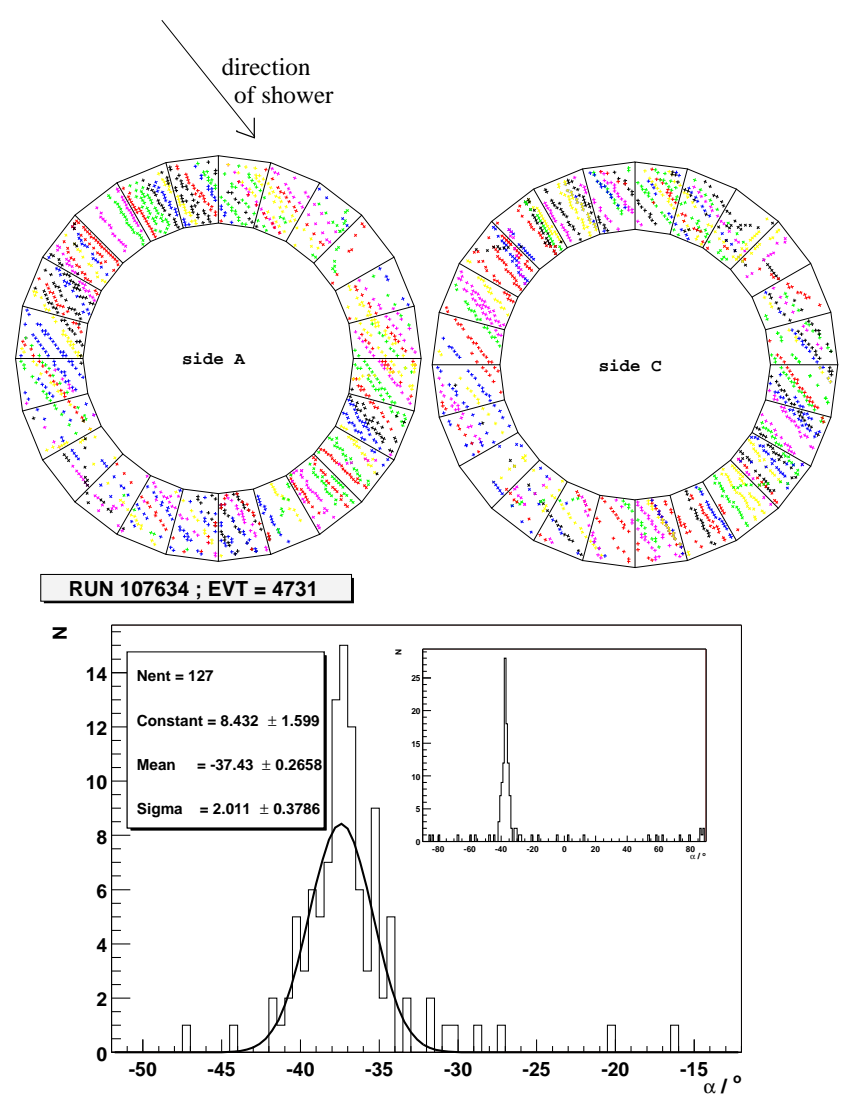

Figure 4: High multiplicity event as seen from HCAL, the angular distribution of reconstructed tracks.

dependency in the $\operatorname{HCAL}(x, y)$ projection assuming zenith angle dependence $\cos ^{3.32} \theta$.

The multiplicity distribution measured in HCAL from selected runs is plotted in Fig. 6 . Energy values marked by arrows in the upper part of the figure box are minimal primary energies of proton induced showers which can still produce events with multiplicities corresponding to the values given on the $x$ axis but not higher.

Additionally to events plotted in Fig. 6 other 7 events with almost saturated HCAL have been detected. For successful reconstruction of those events the information from TPC and muon chambers should be included. Only 2 out of these 7 interesting events have full information from muon chambers, which can be caused by shorter up-time of barrel muon chambers compared to the active time of HCAL cathode readout. Even if the track reconstruction in muon chambers is not available yet for the multi-muon bundles the approximative estimation of multiplicity is done according to the total number of anode hits. Sample of events with information from both HCAL and muon chambers have been studied in order to find the correspondence between the HCAL multiplicity and the number of anodes hit. The conclusion of such preliminary and very approximative analysis gives the value for multiplicity higher than 150 in these 2 exceptional events. 6 events (including 2 with information from muon chambers) are proved to be cosmic events because vacancies in almost saturated HCAL can be fitted by parallel lines. They are assumed to have multiplicity higher than the highest multiplicity reconstructed from data $\left(N_{\mu} \sim 130\right)$. Nothing can be said about the last interesting event because HCAL is saturated fully. 


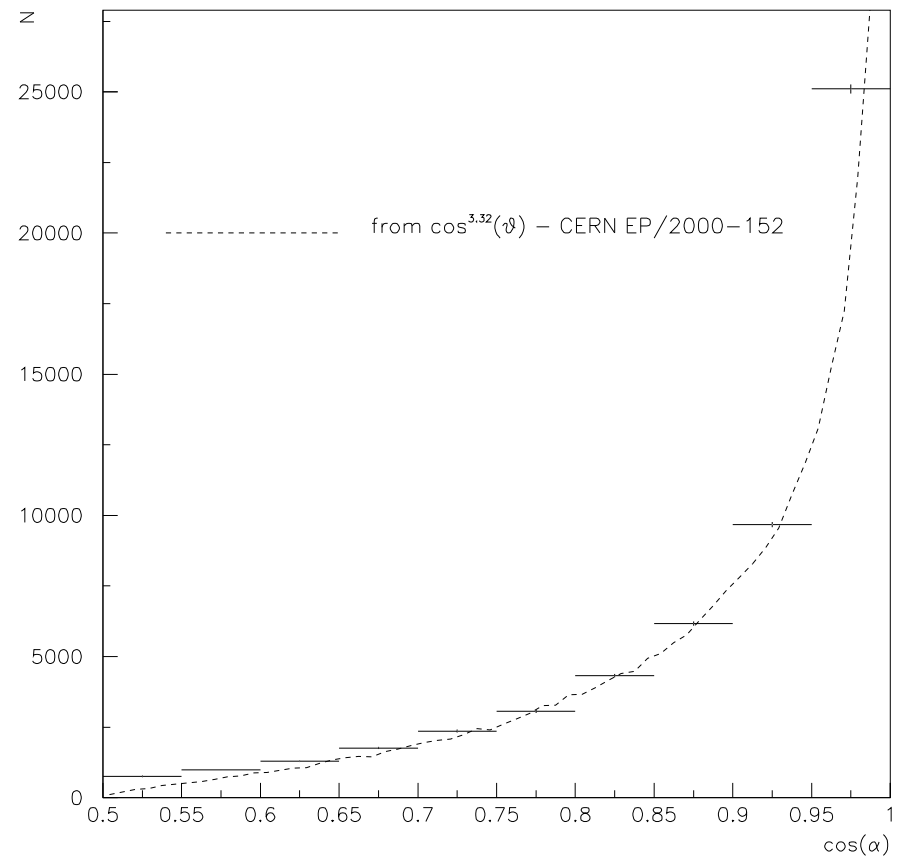

Figure 5: Distribution of $\cos \alpha, \alpha$ is measured in the HCAL projection with respect to the vertical direction.

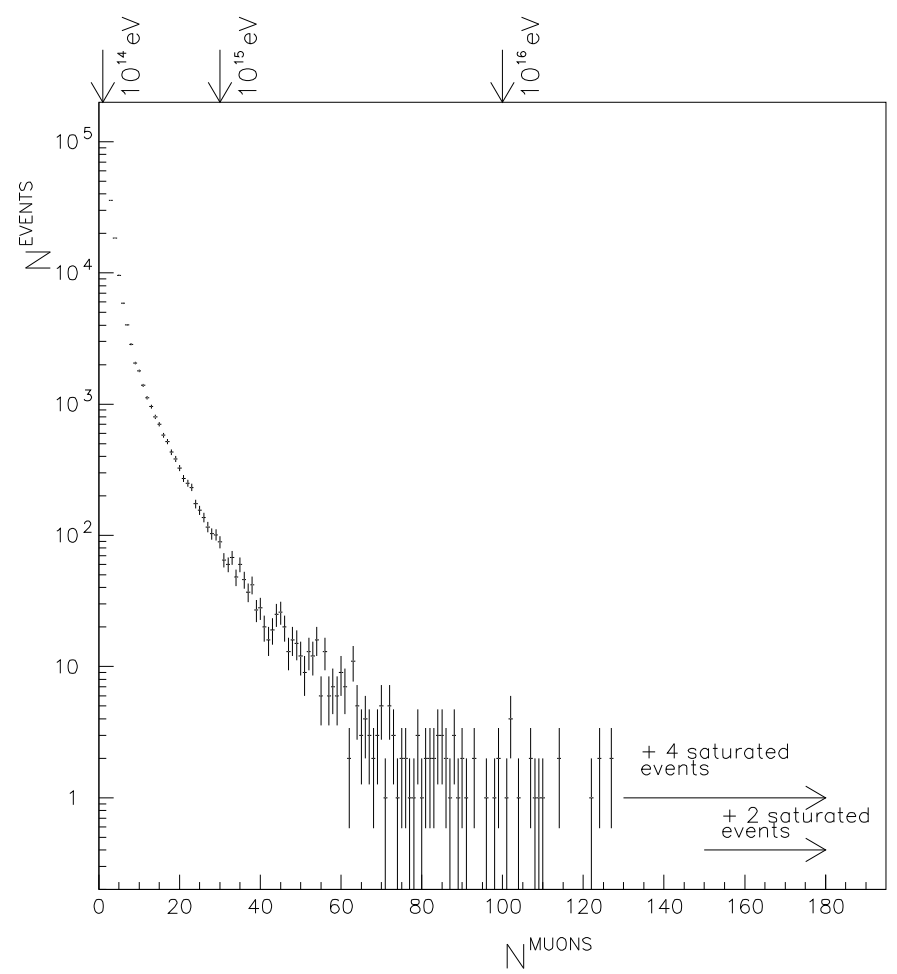

Figure 6: Distribution of muon multiplicity in HCAL. 


\section{Simulation}

In order to be able to simulate the cosmic ray induced showers in the DELPHI environment a new chain of simulation programs has to be set up.

The interaction model ( QGSJET ) is implemented to the CORSIKA [16] simulation package which simulates passage of particles through the atmosphere and shower development. The rock above the DELPHI detector is represented by 5 layers of materials with different densities in a simple GEANT [17] geometry, Fig. 7. Full simulation of the detec-

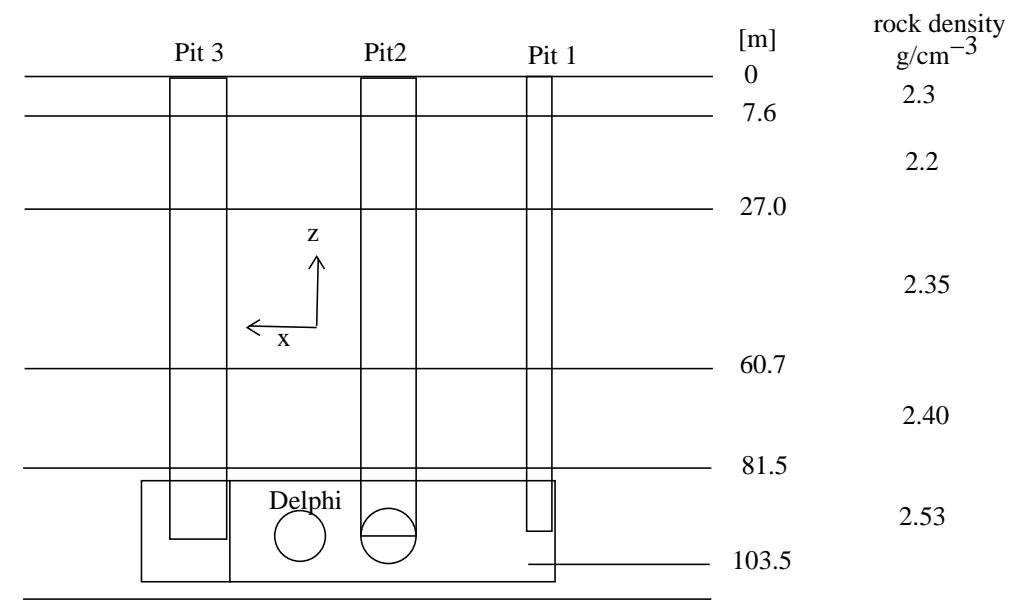

Figure 7: Schematic picture of Geant geometry with 5 layers of rock, and basic structures of experimental cavern.

tor response is provided by the DELSIM simulation package. The test sample of proton showers pointing to the centre of DELPHI apparatus ( 1000 showers with $\left.E=10^{15} \mathrm{eV}\right)$ has been used to check the quality of reconstruction programs. The differences between generated and reconstructed angles of shower directions showed Gaussian peak with $0^{\circ}$ central value and $\sigma \sim 0.5^{\circ}$.

Large data sets are simulated for protons and iron nuclei as primary particles in two energy ranges $10^{14}-10^{16} \mathrm{eV}$ and $10^{16}-10^{18} \mathrm{eV}$. The energy distribution for all simulated data samples was generated with the energy spectrum $E^{-1}$ in order to obtain sufficient statistics at the upper part of the energy spectrum in the range of large multiplicities. Shower centres are smeared over circular area $(R=200 \mathrm{~m})$ around the DELPHI detector. Smaller values of radius $R$ lead to increased fraction of lost events with small muon multiplicities. On the other hand, larger radii imply necessity of large data samples to produce enough events with high muon multiplicities. The value $R=200 \mathrm{~m}$ makes reasonable compromise and ensures fraction of lost events with low multiplicities $(3,4)$ to be smaller than $0.5 \%$.

$\mathrm{MC}$ histograms are weighted according to canonical energy dependence:

for protons with the presence of the 'knee'

$$
f(E) \sim E^{-2.7}, E<210^{6} \mathrm{GeV} \quad f(E) \sim E^{-3.0}, E>210^{6} \mathrm{GeV}
$$

for iron nuclei without the 'knee'

$$
f(E) \sim E^{-2.7}
$$


The energy dependence for iron nuclei flux is taken without the change of spectral index in order to obtain extremal limit for iron initiated multiplicity spectrum. The sensitivity of $\mathrm{MC}$ prediction on the presence of the 'knee' in the spectrum is demonstrated in Fig. 8 for proton primary particles.

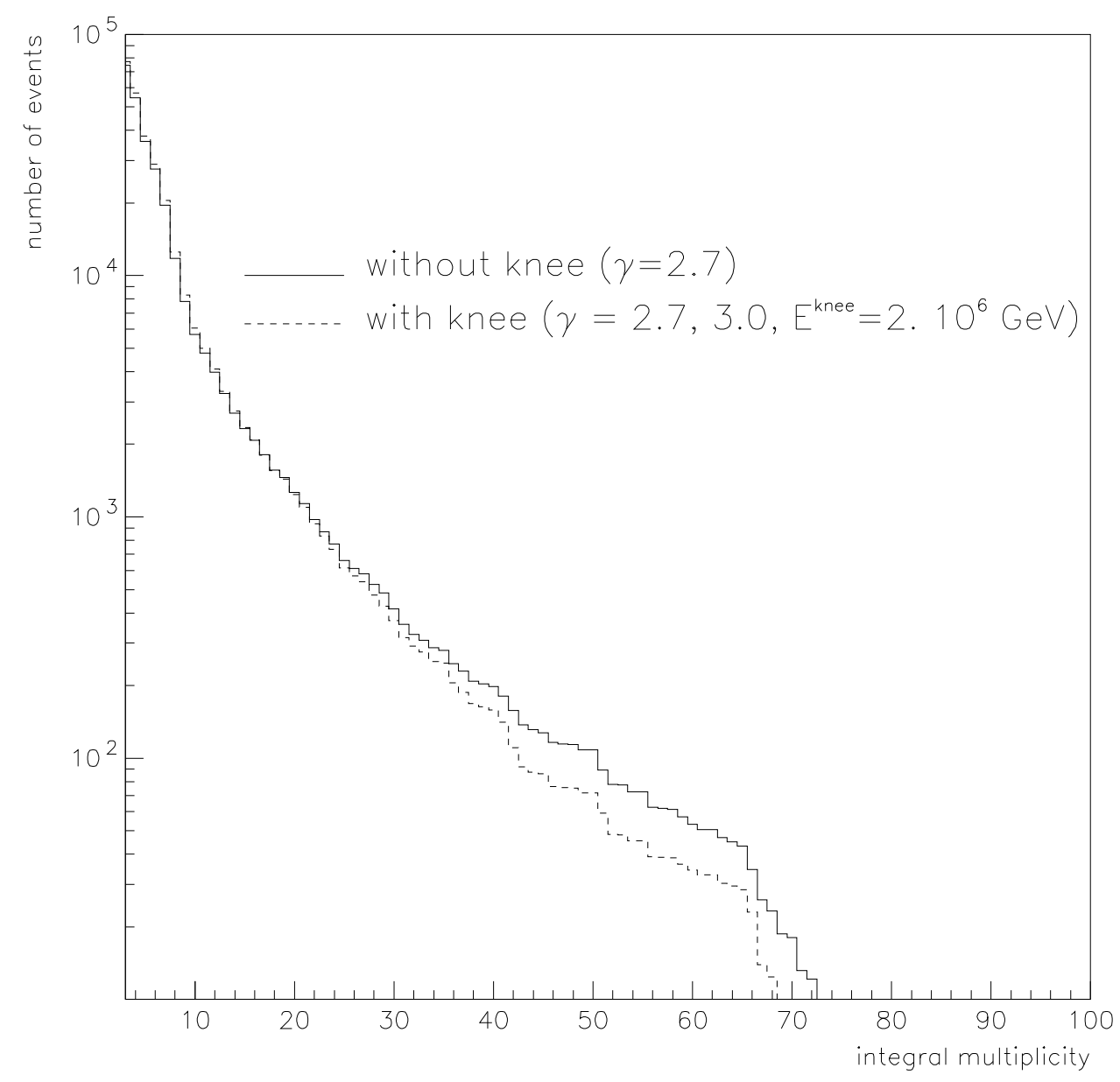

Figure 8: Integral multiplicity for MC protons, with and without 'knee'.

The final MC histograms of muon multiplicities have been normalised to the absolute number of primary particles that would reach circular area (with $R=200 \mathrm{~m}$ ) during data taking time of $1.610^{6} \mathrm{~s}$.

The comparison between MC prediction for protons and iron nuclei as primary particles with the measured data is plotted in Fig. 9. Up to the multiplicity $\sim 80$ the data could be described by composition of these two extreme cases - pure proton and pure iron primary particles. In this region at high multiplicity the necessity to include the heavy component is evident. For even higher multiplicities the MC prediction is not sufficient to reproduce the measurement. The excess of events is apparent similarly as in the analysis [15].

The extreme shower energies $\left(\sim 10^{18} \mathrm{eV}\right)$ in the simulated data sample lead to events with muon multiplicities close to 1000 (inside DELPHI apparatus) in the case the shower 
core is located in the vicinity of the detector. Such high multiplicities are not possible to be reconstructed and these events represent saturated events in the data sample. They appear as the events with multiplicity higher than 130 similarly to the real data. However, these events are so rare, that their total weight is smaller than one and they do not appear in Fig. 9.

The limited range of the energy spectrum used in the simulation $\left(E_{I N C}<10^{18} \mathrm{eV}\right)$ cannot explain the excess of big multiplicity events. This can be illustrated by the events from upper end of the energy spectrum. Only 12 showers with the energy $510^{17} \mathrm{eV}<$ $E_{I N C}<10^{18} \mathrm{eV}$ fall within the circle with radius $200 \mathrm{~m}$ from the DELPHI apparatus centre giving more than 1 muon in the detector. However, only 1.5 events out of these 12 have multiplicity $N_{\mu}>50$ inside DELPHI. Integrated number of events with $E_{I N C}>$ $10^{18} \mathrm{eV}$ falling within $200 \mathrm{~m}$ from DELPHI during $1.610^{6} \mathrm{~s}$ is 0.25 . Thus even increasing the arrival area to include growing lateral shower spread would appear mainly in the lower multiplicity spectrum part.

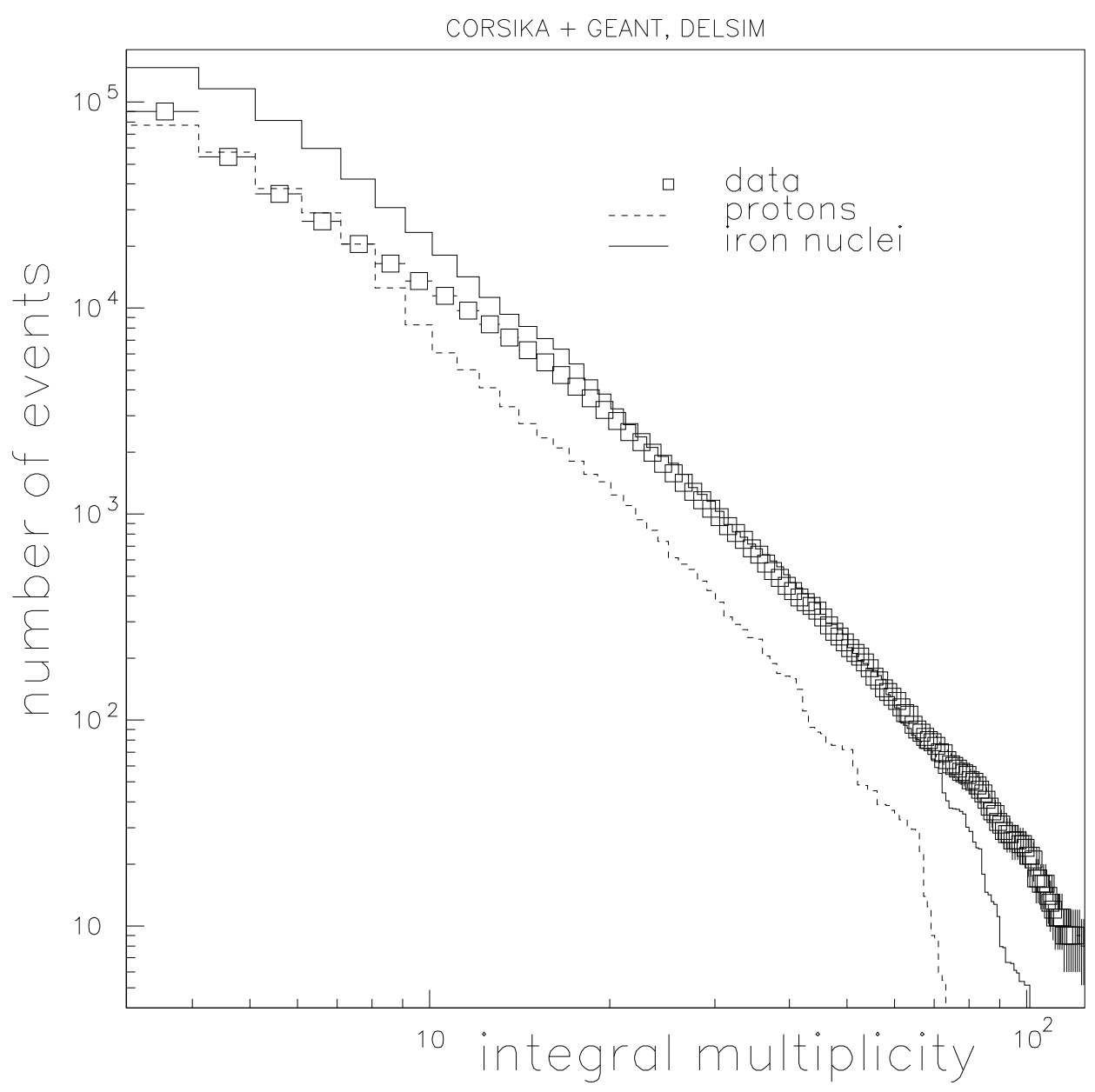

Figure 9: Integral multiplicity for MC protons, irons and the data. 


\section{Conclusions}

The cosmic multi-muon data registered at the DELPHI experiment are analysed. The distribution of muon multiplicities is measured. The MC samples and data are consistent up to the multiplicity of about $\sim 80$. The multiplicity spectrum is sensitive to the primary energy of the impinging particles. However, the multiplicity spectrum is as well sensitive to the chemical composition of the primary radiation. These two variables are correlated and thus with precise independent measurement of the primary energy spectrum one is able to measure the mass spectrum of primary cosmic rays from these underground data. The increased fraction of heavier elements as a function of energy (multiplicity) is necessary to obtain agreement between data and MC prediction. This observation is in agreement with dedicated cosmic ray experiments that study cosmic particles at the knee energies $\left(\sim 10^{15} \mathrm{eV}\right)[18]$. In the presented analysis we have not yet attempted to fit the chemical composition of cosmic rays. The results indicate that the excess of high multiplicity events cannot be explained even by pure flux of iron nuclei. This corroborates the measurements of the COSMO-ALEPH group [15]. However we must study systematic errors and try different interaction models before definite conclusions can be drawn.

The simulation of iron initiated showers in Fig. 9 was done with spectral index -2.7 in the whole simulated energy range. Thus the 'iron' curve represents only the upper limit of the iron initiated spectrum. The inclusion of the knee below $10^{18} \mathrm{eV}$ in the iron nuclei energy spectrum would increase the size of the observed effect (as follows from Fig. 8).

\section{Acknowledgements}

We are greatly indebted to our technical collaborators, to the m embers of the CERN-SL Division for the excellent performance of the LEP collider, and to the funding agencies for their support in building and operating the DE LPHI detector.

We acknowledge in particular the support of Austrian Federal Ministry of Science and Traffics, GZ 616.364/2-III/2a/98, FNRS-FWO, Belgium, FINEP, CNPq, CAPES, FUJB and FAPERJ, Brazil, Czech Ministry of Industry and Trade, GA CR 202/96/0450 and GA AVCR A1010521, Danish Natural Research Council, Commission of the European Communities (DG XII), Direction des Sciences de la Mati'ere, CEA, France, Bundesministerium f" ur Bildung, Wissenschaft, Forschung und Technologie, Germany, General Secretariat for Research and Technology, Greece,

National Science Foundation (NWO) and Foundation for Research on Matter (FOM), The Netherlands, Norwegian Research Council,

State Committee for Scientific Research, Poland, 2P03B06015, 2 P03B1116 and SPUB/P03/178/98,

JNICT-Junta Nacional de Investiga,c ao Cient'ifica e Tecnol'ogica, Portugal,

Vedecka grantova agentura MS SR, Slovakia, Nr. 95/5195/134,

Ministry of Science and Technology of the Republic of Slovenia,

CICYT, Spain, AEN96-1661 and AEN96-1681,

The Swedish Natural Science Research Council, 
Particle Physics and Astronomy Research Council, UK, Department of Energy, USA, DE-FG02-94ER40817.

\section{References}

[1] T.Antoni at al.: Electron, Muon and Hadron Lateral Distributions Measured in AirShowers by the KASCADE Experiment, Astropart. Phys. 14,(2001) 245-260.

[2] MACRO Collaboration, M. Ambrosio et al.: Phys. Rev. D 52,(1995) 3793.

[3] SOUDAN Collaboration, Internal Report No.PDK-435, 1990 (unpublished).

[4] C.Castagnoli and O.Saavedra, Nuovo Cimento C 9,(1986) 111.

[5] NUSEX Collaboration, M. Aglietta et al.: Proceedings of the topical seminar 'Astrophysics and Particle Physics', San Miniato, Italy, 1989, edited by G. Castellini et al. [Nucl. Phys. B(Proc. Suppl.) 14B, (1990) 193.]

[6] LVD Collaboration, M. Aglietta et al.: Astropart. Phys. 3,(1995) 311.

[7] Fréjus Collaboration, W. Rhode et al.: Astropart. Phys. 4,(1996) 217.

[8] E.V. Bugaev et al.: Phys. Rev. D 58,(1998) 1.

[9] Yu.M. Andreyev, A.E. Chudakov, V.I. Gurentsov and I.M. Kogai: Proceedings of the 21st International Cosmic Ray Conference, Adelaide, Australia, January 6-19, 1990, edited by R.J. Protheroe (Dept. of Physics and Mathematical Physics, Univ. of Adelaide, Northfield, South Australia, 1990), Vol. 9, p. 301.

[10] N. Ito (for the KGF Collaboration): Proceedings of the International Symposium on Underground Physics Experiment, Tokyo, Japan, 1990, edited by K. Nakamura (ICRR, Tokyo, 1990), p. 101.

[11] N.N. Kalmykov and S.S. Ostapchenko: Yad. Fiz. 56 (1993), 105. N.N. Kalmykov and S.S. Ostapchenko: Phys. At. Nucl. 56 (1993), 346. N.N. Kalmykov, S.S. Ostapchenko and A.I. Pavlov: Bull. Russ. Acad. Sci. (Physics) 58 (1994), 1966.

[12] A.A. Watson: Proc. '25th International Cosmic Ray Conference', Durban, South Africa, 1997, Vol. 8, p257.

[13] By KASCADE Collaboration (T. Antoni et al.) Astroparticle Physics 162452002.

[14] J.Ridky, V.Vrba, J.Chudoba: ECTANA, User's Guide, DELPHI 99-181 TRACK 96.

[15] V. Avati et al, CERN EP/2000-192.

[16] D. Heck et al.: CORSIKA: A Monte Carlo Code to Simulate Extensive Air Showers, Forschungszentrum Karlsruhe, Technik und Umwelt, Wissenschaftliche Berichte FZKA 6019, 1998.

[17] R.Brun et al.: GEANT CERN Program Library Writeup W5013, 1993. 
[18] K-H. Kampert et al.: Contribution to '26th International Cosmic Ray Conference', OG 1.2.11, Salt Lake City, Utah, August 17-25, 1999. 